\title{
Blue stragglers in open clusters ${ }^{\star}$
}

\section{NGC 7789}

\author{
D. Schönberner ${ }^{1}$, S. M. Andrievsky ${ }^{1,2,3}$, and J. S. Drilling ${ }^{4}$ \\ 1 Astrophysikalisches Institut Potsdam, An der Sternwarte 16, 14482 Potsdam, Germany \\ e-mail: DeSchoenberner@aip.de \\ 2 Department of Astronomy, Odessa State University, Shevchenko Park, 65014, Odessa, Ukraine \\ 3 Isaac Newton Institute of Chile, Odessa Branch, Chile \\ e-mail: scan@deneb.odessa.ua \\ 4 Louisiana State University, Department of Physics and Astronomy, Baton Rouge, Louisiana 70803, USA \\ e-mail: Drilling@baton.phys.1su.edu
}

Received 8 September 2000 / Accepted 7 November 2000

\begin{abstract}
We performed for the first time a detailed LTE spectroscopic study of a sample of blue straggler stars in the moderately old open cluster NGC 7789. For eight stars the parameters and abundances of several elements were determined. The cluster members show a remarkable surface magnesium deficiency which is quite unusual for late B - early A stars. Iron and titanium abundances are in agreement with other photometric and spectroscopic estimates of the NGC 7789 metallicity. All the confirmed blue stragglers have rather low projected rotational velocities (with one exception for K88, $v \sin i=80 \mathrm{~km} \mathrm{~s}^{-1}$ ).
\end{abstract}

Key words. stars: open clusters and associations: individual: NGC 7789 - stars: blue stragglers - stars: abundances

\section{Introduction}

The moderately old open cluster NGC 7789 is among those having very rich blue-straggler populations. Ahumada \& Lapasset (1995) give a cluster age of $1.6 \mathrm{Gyr}$, and the number of suspected blue-straggler candidates is reported to be 25. Despite this large blue-straggler population, no quantitative abundance analysis has been performed so far.

Twenty years ago NGC 7789 with its blue stragglers became a paradigm to explain the existence of such stars by assuming ad hoc additional internal mixing which extends the main sequence phase (Saio \& Wheeler 1980). Such a putative mechanism was later discussed by Schönberner \& Napiwotzki (1994) who determined accurate effective temperatures and gravities for several blue stragglers in NGC 7789. These authors have shown that the existence of blue stragglers does not result from

Send offprint requests to: S. M. Andrievsky

* Based on the spectra collected at Kitt Peak National Observatory, operated by the Association of Universities for Research in Astronomy, Inc., under contract with the National Science Foundation, and at the German-Spanish Astronomical Center, Calar Alto, operated by the Max-Plank Institute for Astronomy, Heidelberg, jointly with the Spanish National Commission for Astronomy. internal mixing processes, but that they rather evolve like normal stars.

In recent years interest in the NGC 7789 blue stragglers (and in blue stragglers in general) has waned because of the failure to find any spectroscopic signature unique to this phenomenon.

Our third paper from this series is devoted to a detailed spectroscopic investigation of blue-straggler candidates in NGC 7789 .

\section{Program stars and observations}

We have been able to secure spectrograms of a total of 8 blue-straggler candidates at two different observatories (Kitt Peak National Observatory - KPNO, and Calar Alto Observatory - CA). Some essential information about our targets and details of their observation are given in Table 1. Additional information about the stars listed in the table can be also found in the WEBDA data base (Mermilliod 1999). The well investigated star $\alpha$ Lyr (Vega) has been chosen as a reference star in order to control our method of the analysis.

KPNO spectra were obtained during October 1-3, 1985, with the 4-m telescope equipped with an echelle spectrograph and CCD camera (resolution $0.12 \AA / \mathrm{px}$, 
Table 1. Data for program stars

\begin{tabular}{|c|c|c|c|c|c|c|}
\hline K & GET & $V$ & $B-V$ & $\mathrm{MP}$ & Region, $\AA$ & Instrument \\
\hline K88 & 2873 & 13.10 & 0.38 & 0.98 & $4220-4525$ & KPNO \\
\hline K316 & 5569 & 13.79 & 0.36 & 0.98 & $4220-4525$ & KPNO \\
\hline K371 & 5951 & 12.95 & 0.36 & 0.98 & $\begin{array}{c}4220-4525 \\
\mathrm{H} \gamma, \mathrm{H} \beta\end{array}$ & $\begin{array}{c}\text { KPNO } \\
\text { CA }\end{array}$ \\
\hline K409 & 6268 & 12.98 & 0.31 & 0.53 & $\begin{array}{c}4220-4525 \\
\mathrm{H} \gamma\end{array}$ & $\begin{array}{c}\mathrm{KPNO} \\
\mathrm{CA}\end{array}$ \\
\hline K677 & 8404 & 11.16 & 0.16 & 0.53 & $\begin{array}{c}4220-4525 \\
4450-4585, \mathrm{H} \beta\end{array}$ & $\begin{array}{c}\mathrm{KPNO} \\
\mathrm{CA}\end{array}$ \\
\hline K746 & 8804 & 12.74 & 0.39 & 0.98 & $\begin{array}{c}4220-4525 \\
\mathrm{H} \gamma\end{array}$ & $\begin{array}{c}\mathrm{KPNO} \\
\mathrm{CA}\end{array}$ \\
\hline K1211 & 13273 & 11.55 & 0.18 & 0.97 & $\begin{array}{c}\mathrm{H} \gamma, \mathrm{H} \beta \\
4450-4580\end{array}$ & $\begin{array}{l}\mathrm{CA} \\
\mathrm{CA}\end{array}$ \\
\hline K1270 & 14054 & 13.46 & 0.46 & 0.98 & $4220-4525$ & KPNO \\
\hline Vega & & 0.06 & & & $4220-4525$ & KPNO \\
\hline
\end{tabular}

K - Küstner (1923) number; GET - Gim et al. (1998a) number; MP - membership probability as determined by McNamara \& Solomon (1981).

$\mathrm{S} / \mathrm{N}$ ratio varying from approximately 30 to 80 at the continuum level, except for Vega). These spectrograms were extracted from the frames in the usual manner by using the IRAF package ${ }^{1}$. All spectra consisted of nine (not overlapping) orders, each covering approximately $30 \AA$. Because of the small sizes of the observed bands we met some problems with the correct continuum placement in the vicinity of the $\mathrm{H} \gamma$ line. To avoid the problems that could be caused by the wrong continuum placement, the two adjacent orders from the spectrum of each star ranging from $4320 \AA$ to $4390 \AA$ were discarded in the further analysis.

The CA spectral material was obtained during two runs in January and September 1986 with the 2.2-m telescope equipped with a Coudé spectrograph (resolution $0.26 \AA / \mathrm{px}, \mathrm{S} / \mathrm{N}$ ratio about $40-70$ ). These observations were aimed at getting reliable profiles of the hydrogen lines $\mathrm{H} \gamma$ and $\mathrm{H} \beta$. The spectral regions cover approximately $130 \AA$ each.

The final spectra were treated using the DECH20 spectra processing package (Galazutdinov 1992).

\section{Stellar parameters}

The effective temperatures were estimated using the Strömgren photometric indices (available for the program stars through the WEBDA data base, see Mermilliod 1999) by means of the numerical code written by T.T. Moon (based on the grid published in Moon \& Dworetsky 1985) and modified by Napiwotzki (1994). In the WEBDA data base, however, the $\beta$-index is given for only two of our program stars. For the remaining ones we used

\footnotetext{
1 Distributed by NOAO operated by the Association of Universities for Research in Astronomy, Inc., under contract to the NSF.
}

either the measurements given in the paper of Schönberner \& Napiwotzki (1994), or obtained by Schönberner (unpublished) with the same technique as explained in Schönberner \& Napiwotzki (1994).

In cases where $\mathrm{H} \gamma$ and $\mathrm{H} \beta$ profiles were available (cf. Table 1) the gravities were specified by matching observed and calculated profiles. The profiles of the hydrogen lines were calculated with the help of the SYNSPEC code (Hubeny et al. 1994). For K88, K316 and K1270 hydrogen profiles are not available, and we used gravities determined by photometry. However, for K316 and K1270 the photometrically determined gravities appeared to be inconsistent with their positions in the HR diagram (see Fig. 4 from Breger 1982). For the less evolved star, K316, we adopted $\log g=4.0$, and for K1270 $\log g=3.5$. Note that these adopted gravities differ $\approx 0.4$ dex from the photometrically estimated ones, but this difference has a small influence on the derived abundances in the temperature region in question.

The finally adopted effective temperatures and gravities are collected in Table 2. Note that for K409, K677, K1211 and Vega the values given in Schönberner \& Napiwotzki (1994) were used.

Projected rotational velocities were determined by matching observed and calculated profiles of the hydrogen and/or metallic lines (mainly Mg II) and are also listed in Table 2 .

\section{Elemental abundances}

The atmosphere models of Kurucz (1991) and oscillator strengths for the lines of interest from the VALD data base were used to determine individual elemental abundances in conjunction with the LTE spectrum synthesis method (SYNSPEC, Hubeny et al. 1994). For all the program blue stragglers we adopted the microturbulent velocity 
Table 2. Parameters of program stars

\begin{tabular}{lccc}
\hline Knum & $T_{\text {eff }}(\mathrm{K})$ & $\log g$ & $v \sin i\left(\mathrm{~km} \mathrm{~s}^{-1}\right)$ \\
\hline K88 & 8900 & 3.7 & 80 \\
K316 & 8950 & 4.0 & 25 \\
K371 & 8500 & 3.7 & 45 \\
K409 & 9480 & 4.2 & 140 \\
K677 & 10680 & 3.6 & 40 \\
K746 & 8600 & 3.5 & 20 \\
K1211 & 12630 & 3.6 & 30 \\
K1270 & 8300 & 3.5 & 25 \\
\hline Vega & 9500 & 4.0 & 22 \\
\hline
\end{tabular}

$V_{\mathrm{t}}=3 \mathrm{~km} \mathrm{~s}^{-1}$ (the same value as adopted in Andrievsky et al. 2000 - Paper II), while for Vega we used the literature value $V_{\mathrm{t}}=2 \mathrm{~km} \mathrm{~s}^{-1}$ (see, e.g. Sadakane \& Nishimura 1981). For the sake of completeness, we also calculated the abundances with the smaller value of $V_{\mathrm{t}}=0.6 \mathrm{~km} \mathrm{~s}^{-1}$ recommended by Adelman \& Gulliver (1990). Note that our implementation of the SYNSPEC code enables one to calculate only the spectra of chemical elements with $Z \leq 30$, therefore, the lines of the heavier species (if observed) were ignored. The steps of the analysis mentioned above are described in more detail in Paper II.

There is much evidence that Vega is a mild metal deficient star, therefore, we used the atmosphere model selected from the grid with $[\mathrm{A}]=-0.5$ for its spectrum synthesis.

Elemental abundances for Vega and our program blue stragglers are given in Tables 3 and 4 . For Vega we also give a comparison with the most recent study by Hill (1995) also based on the spectrum synthesis technique. It should be stressed that with this study we did not aim to enlarge the number of precise and detailed spectroscopic investigations of Vega. Our main reason was to have some external indication of the reliability of the analysis based on our homogeneous spectroscopic material. Although the number of lines used is limited, the agreement in the derived abundances with Hill (1995) is satisfactory.

All of our high-probability proper-motion members show a strong deficiency of magnesium and scandium. For example, in Figs. 1 and 2 the synthetic and observed spectra for program stars in the vicinity of the MgII $4481 \AA$ line are shown. The synthetic spectra were calculated with the parameters listed in Table 2 and magnesium abundances for individual stars from Tables 3 and 4 .

The detected magnesium deficiency for blue straggler stars cannot be removed by any reasonable changes in the atmospheric parameters, because within the temperature region $8000 \mathrm{~K}-10000 \mathrm{~K}$, the magnesium line $4481 \AA$ appears to be practically insensitive to temperature and gravity variations. This was also mentioned by Holweger, Gigas \& Steffen (1986) who performed a qualitative search for abundance indicators in early A stars which are both temperature and gravity insensitive. As an example, we have calculated the magnesium abundance for K1270
Table 3. Elemental abundances for Vega

\begin{tabular}{cccccc}
\hline \multicolumn{4}{c}{ Present Paper } & & Hill (1995) \\
\hline \multicolumn{4}{c}{$0.6 \mathrm{~km} \mathrm{~s}^{-1}$} & \multicolumn{2}{c}{$2.0 \mathrm{~km} \mathrm{~s}^{-1}$} \\
\hline $\mathrm{El}$. & {$[\mathrm{El} / \mathrm{H}]$} & {$[\mathrm{El} / \mathrm{H}]$} & $\sigma$ & $\mathrm{N}$ & {$[\mathrm{El} / \mathrm{H}]$} \\
\hline $\mathrm{Mg}$ & -0.10 & -0.26 & - & 1 & -0.27 \\
$\mathrm{Ca}$ & -0.63 & -0.73 & - & 1 & -0.47 \\
$\mathrm{Sc}$ & -1.27 & -1.41 & - & 1 & - \\
$\mathrm{Ti}$ & -0.30 & -0.64 & 0.09 & 7 & -0.46 \\
$\mathrm{Cr}$ & -0.25 & -0.34 & - & 1 & -0.48 \\
$\mathrm{Fe}$ & -0.18 & -0.46 & 0.11 & 7 & -0.54 \\
\hline
\end{tabular}

assuming temperature and gravity uncertainties of about $\pm 500 \mathrm{~K}$ and \pm 0.5 dex respectively. The resulting changes in the abundance appeared to be negligible, and even smaller for temperatures higher than $8500 \mathrm{~K}$. The response of the magnesium abundance (as derived from the Mg II $4481 \AA$ line) caused by the parameter variations is given in Table 5 . The $\Delta$ means the difference between the magnesium abundance $[\mathrm{Mg} / \mathrm{H}]$ derived for K1270 with its basic model parameters (see Table 2 ) and with varied parameters (e.g., $7750 / 4.0 / 3.0$ denotes model with $T_{\text {eff }}=$ $7750 \mathrm{~K}, \log g=4.0$ and $\left.V_{\mathrm{t}}=3.0 \mathrm{~km} \mathrm{~s}^{-1}\right)$.

For the iron-group ( $\mathrm{Ti}, \mathrm{Fe})$, the abundances for the blue stragglers do not differ from those determined for other stars in NGC 7789. Tiede et al. (1997) estimated the metallicity of NGC 7789 by IR photometry of the giant branch to be $[\mathrm{Fe} / \mathrm{H}]=-0.62$. Recently, Vallenary et al. (2000) revised this value by an improved method using new IR photometry and found $[\mathrm{Fe} / \mathrm{H}]=-0.25 \pm 0.11$. Friel \& Janes (1993) carried out low-resolution spectroscopy for several giants and obtained a mean metallicity of $[\mathrm{Fe} / \mathrm{H}]=-0.26 \pm 0.06$. Pilachowski (1985) performed a high-resolution spectroscopic investigation of six giant stars and found $[\mathrm{Fe} / \mathrm{H}]=-0.1 \pm 0.2$, in excellent agreement with our mean iron abundance $(-0.16 \mathrm{dex})$.

As to other elements, there is only one indication in the literature that the relative-to-solar abundances of atomic species like $\mathrm{Ca}, \mathrm{Sc}$, Ti, etc. scatter only slightly about that of iron (Pilachowski 1985). Our results for titanium and chromium agree very well with those of Pilachowski, that of calcium only marginally. The only really discrepant case is for scandium where our blue stragglers appear to be deficient between a factor of 10 to 100 . It is known, however, that A-type stars very often show peculiarities of certain elements (see Sect. 5.2).

\section{Discussion}

\subsection{Cluster membership}

The membership probabilities for our blue-straggler candidates in NGC 7789 have been determined by several investigations:

Photometry, polarimetry and proper motion:

Pendl (1975), McNamara (1980), Twarog \& Tyson (1985), Manteiga et al. (1991), Breger (1982). 
Table 4. Relative elemental abundances $[\mathrm{El} / \mathrm{H}]$ for program stars in the field of NGC 7789

\begin{tabular}{|c|c|c|c|c|c|c|c|}
\hline Star & $\mathrm{He}$ & $\mathrm{Mg}$ & $\mathrm{Ca}$ & $\mathrm{Sc}$ & $\mathrm{Ti}$ & $\mathrm{Cr}$ & $\mathrm{Fe}$ \\
\hline K88 & & $-0.70(-, 1)$ & $-0.25(-, 1)$ & $-1.11(-, 1)$ & $-0.28(0.18,5)$ & & $-0.12(0.20,5)$ \\
\hline K316 & & $-1.04(-, 1)$ & $-0.53(-, 1)$ & $-1.31(0.18,4)$ & $-0.41(0.19,3)$ & $+0.09(-, 2)$ & $-0.23(0.19,11)$ \\
\hline K371 & & $-0.20(-, 1)$ & & $-0.77(-, 1)$ & & & $-0.13(0.23,4)$ \\
\hline K409 & & $+0.00(-, 1)$ & & & & & \\
\hline K677 & $+0.07(-, 1)$ & $+0.16(0.18,5)$ & & & $-0.24(0.22,6)$ & & $-0.08(0.17,7)$ \\
\hline K746 & & $-0.64(-, 1)$ & $-0.14(0.17,4)$ & $-0.92(0.25,5)$ & $-0.23(0.19,15)$ & $+0.03(0.16,4)$ & $-0.13(0.19,13)$ \\
\hline K1211 & $-1.00(-, 1)$ & $-1.04(-, 1)$ & & & & & $-0.10(0.23,4)$ \\
\hline K1270 & & $-1.14(-, 1)$ & $-0.71(0.29,4)$ & $-2.03(0.19,5)$ & $-0.39(0.24,11)$ & $+0.00(0.18,4)$ & $-0.20(0.18,15)$ \\
\hline
\end{tabular}

Table 5. Parameter variation and Mg abundance changes for K1270

\begin{tabular}{ccccccc}
\hline & $7750 / 3.0 / 3.0$ & $7750 / 4.0 / 3.0$ & $8750 / 3.0 / 3.0$ & $8750 / 4.0 / 3.0$ & $8300 / 3.6 / 2.5$ & $8300 / 3.6 / 3.5$ \\
\hline$\Delta$ & -0.12 & +0.18 & -0.05 & +0.05 & +0.09 & -0.05 \\
\hline
\end{tabular}

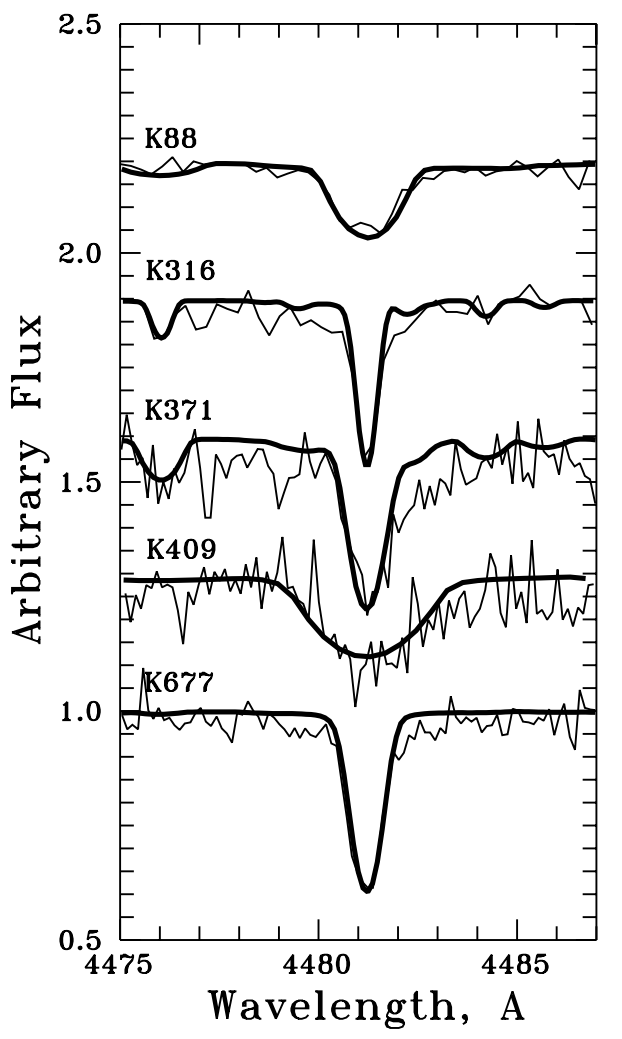

Fig. 1. Observed (thin line) and calculated (thick line) fragment of the spectra in the vicinity of Mg II $4481 \AA$ line

Radial velocities: Strom \& Strom (1970), Stryker \& Hrivnak (1984), Drilling \& Schönberner (1987), Manteiga et al. (1989), Milone \& Latham (1994). Spectroscopy: Schönberner \& Napiwotzki (1994).

In discussing the cluster membership problem we exclusively rely only on the most stringent criteria, viz. proper motion, radial velocity and spectroscopy. Based on the proper motion study of McNamara (1980) and Pendl (1975) we selected those objects from McNamara's lists

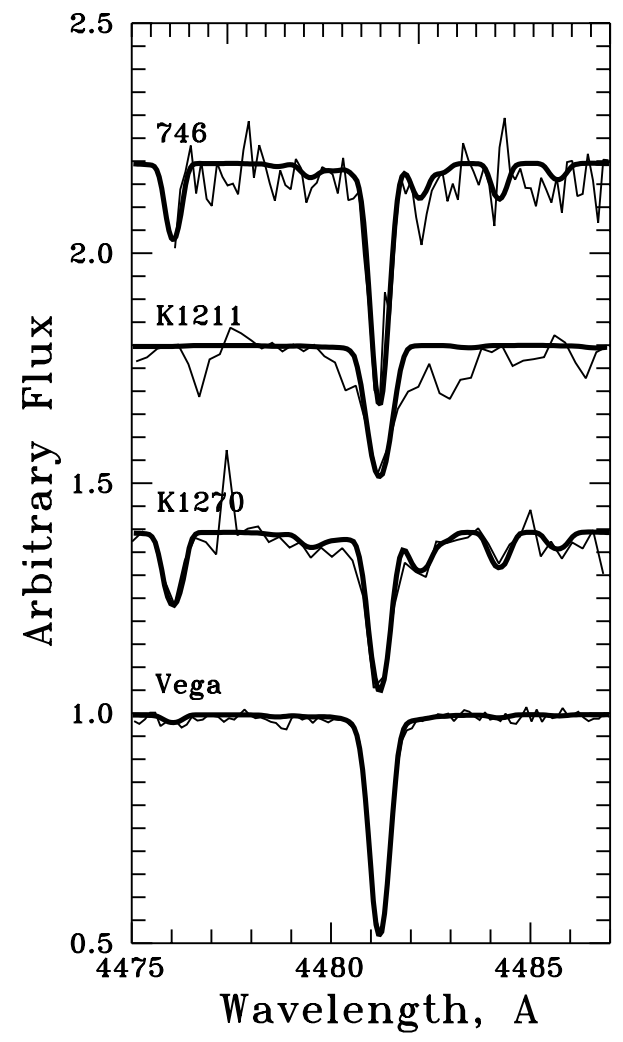

Fig. 2. Same as Fig. 1

of blue straggler candidates with at least one measurement of the radial velocity. Instead of reporting the qualitative judgements of the respective authors, we retrieved the original data and listed them in Table 6 .

We also revised the radial velocities for several blue stragglers previously analysed by Drilling \& Schönberner (1987). We used the KPNO spectra and applied the very accurate method of line mirroring. The results are given in the corresponding column of Table 6 . We checked the method by applying it to our reference star Vega. From 14 lines of its KPNO spectrum we derived the mean value 
of $V_{\mathrm{r}}=-12.7 \pm 0.6 \mathrm{~km} \mathrm{~s}^{-1}$, in good agreement with the recommended value of $-13.9 \mathrm{~km} \mathrm{~s}^{-1}$ (SIMBAD data base).

The problem of the determination of true, i.e. physical, cluster memberships is somewhat delicate and prone to personal opinions. The necessary condition for an object to be a (physical) cluster member is the agreement of its proper motion and radial velocity with those of the cluster.

Spectroscopy, as performed by Schönberner \& Napiwotzki (1994), serves as a final criterion because it provides a distance information. If, however, spectroscopy indicates membership, it must be supplemented by the proper motion and radial velocity data in order to distinguish between the physical members and field interlopers (see the case of K677 in Table 6).

The membership assignments of Table 6 are based on the philosophy outlined above. Note that in the two cases (K409 and K1211) where spectroscopy indicates non-membership, this statement is supported by the radial velocity measurements. Altogether we consider 11 of the 26 blue-straggler candidates to be the members of NGC 7789 .

\subsection{Abundance peculiarities}

The fact that the blue stragglers in NGC 7789 display abundance anomalies for some chemical elements, which are not seen in the convectively well-mixed cluster giants, indicates that these anomalies are purely surface phenomena. All investigated blue stragglers fall in the domain of late B - early/mid A-type stars. It is well known that stars of these spectral types demonstrate a great variety of chemical peculiarities. Our investigated blue stragglers possess projected rotational velocities which are lower than is expected for their spectral classes. It is therefore interesting to compare their abundance anomalies (for the confirmed cluster members only, see Sect. 5.1) with those of chemically peculiar stars.

Normal A-type stars. Numerous studies of sharp-lined late B - early A-type stars were performed by Adelman with co-authors (see, e.g. Caliskan \& Adelman 1997 and references therein). They showed that some of these stars have solar-like elemental distributions. For example, Caliskan \& Adelman (1997) and Adelman (1999) provide elemental abundances for 17 stars (without Vega and the $\lambda$ Boo type star 29 Cyg). From this sample one can derive a mean magnesium abundance (from the $\mathrm{Mg}$ II lines only) of $[\mathrm{Mg} / \mathrm{H}]=$ $-0.11 \pm 0.19$. This value is significantly larger than the magnesium abundance of our blue stragglers. In Fig. 3 we compare their abundance distribution (that of Vega from Table 3 included) with data from the literature on A stars possessing small projected rotational velocities. As one can see from Fig. 3 the blue straggler

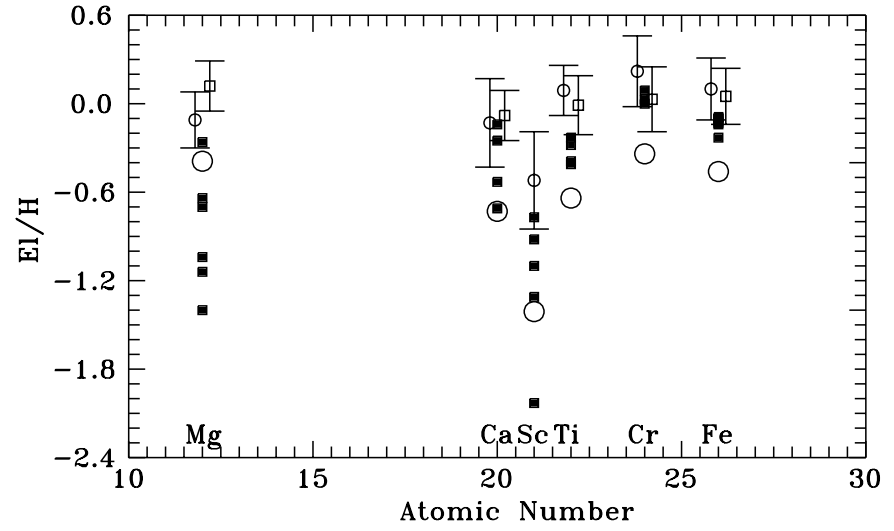

Fig. 3. Comparison of different abundance distributions. Open circles: mean values of "normal" A-type stars from Caliskan \& Adelman (1997) and Adelman (1999); open squares: mean values from Hill (1995); filled squares: individual values of the blue stragglers investigated in this paper; large open circles: our data for Vega. Note that the literature values are horizontally shifted by \pm 0.2 , and that the typical error $(2 \sigma)$ is indicated

abundance distribution follows the general trend, with the exception of $\mathrm{Mg}$ and $\mathrm{Sc}$ which appear to be depleted.

Am stars. According to the classical definition, Am stars have apparent surface underabundances of $\mathrm{Ca}$ and $\mathrm{Sc}$ (see Cayrel et al. 1991). This is also the case for the blue stragglers studied here. Am stars are, however, also characterized by a moderate overabundance of the iron-group and heavier elements, but this is not seen in NGC 7789 (we recall that we did not investigate elements heavier than iron). Also, the remarkable magnesium deficiency of the NGC 7789 blue stragglers is not reported for Am stars.

Ap stars. Ap stars show even more pronounced abundance anomalies, especially for heavy species. On the other side, magnesium, calcium and scandium can often be substantially depleted. With the spectral material at our disposal we are not able to decide whether our blue-straggler sample contains Ap stars. For example, the Sr II $4215 \AA$ classification feature is not covered by the observed spectral region, although the rather strong lines of Zr II 4496.96 $\AA$ and Y II 4398.02 $\AA$ are seen in the spectra of K746 and K1270. It should be also mentioned that Stryker \& Hrivnak (1984) noted that K1211 shows relatively strong Si II lines and an absence of the helium lines (the latter is in agreement with our result on helium abundance in this star). The above mentioned authors classified K1211 as Ap(Si) star.

Mg II $4481 \AA$ weak stars. The weak Mg II $4481 \AA$ line is an inherent feature of some A stars, as described by Abt \& Morrell (1995). These authors consider them as a mild version of the $\lambda$ Boo-type stars (see the next item). Using the data of Abt \& Morrell (1995) we plot the equivalent widths of the magnesium line vs. spectral class for normal A-type stars, 
Table 6. Radial velocities (in $\mathrm{km} \mathrm{s}^{-1}$ ) and memberships of blue stragglers in NGC 7789. The cluster velocity based on red giants is $V_{\mathrm{r}}=-55 \mathrm{~km} \mathrm{~s}^{-1}$ (Gim et al. 1998b)

\begin{tabular}{|c|c|c|c|c|c|c|c|c|c|c|}
\hline \multicolumn{4}{|c|}{ Star number } & \multicolumn{4}{|c|}{ Radial velocity } & \multicolumn{3}{|c|}{ Spectroscopy } \\
\hline M & $\mathrm{K}$ & SS70 & SH84 & $\mathrm{DS} 87(\mathrm{CA})$ & DS87(KPNO) & MPMR89 & ML94 & This work & SN94 & | \\
\hline 257 & 2 & - & - & - & - & - & $\mathrm{N}$ & - & - & $\mathrm{N}$ \\
\hline 317 & 68 & - & - & - & - & - & $\mathrm{N}$ & - & - & $\mathrm{N}$ \\
\hline 325 & 88 & - & - & - & - & - & $\mathrm{Y}$ & $-58 \pm 4(6)$ & - & $M^{*}$ \\
\hline 377 & 168 & -21 & - & - & - & - & $\mathrm{U}$ & - & - & $\mathrm{U}$ \\
\hline 389 & 192 & - & - & - & - & - & $\mathrm{U}$ & - & - & $\mathrm{U}$ \\
\hline 396 & 197 & -31 & - & - & - & - & $\mathrm{N}$ & - & - & $\mathrm{N}$ \\
\hline 419 & 234 & -26 & - & - & - & - & - & - & - & $\mathrm{N}$ \\
\hline 460 & 282 & - & -47 & -54 & - & -43 & M & - & M & $\mathrm{U}$ \\
\hline 482 & 316 & - & - & - & -58 & - & $\mathrm{Y}$ & $-59 \pm 3(15)$ & - & M \\
\hline 502 & 342 & -45 & -51 & -56 & - & -51 & $\mathrm{U}$ & - & M & M \\
\hline 500 & 349 & -34 & - & - & - & - & - & - & - & $\mathrm{N}$ \\
\hline 518 & 371 & -56 & -56 & -55 & -65 & -58 & $\mathrm{Y}$ & $-56 \pm 5(6)$ & - & M \\
\hline 543 & 409 & -20 & -45 & -27 & -29 & - & $\mathrm{N}$ & $-28(1)$ & $\mathrm{N}$ & $\mathrm{N}$ \\
\hline 574 & 453 & -41 & -52 & -41 & - & -71 & $\mathrm{U}$ & - & M & M \\
\hline 747 & 677 & - & -37 & -27 & -23 & -18 & $\mathrm{~N}$ & $-17 \pm 4(14)$ & M & $\mathrm{N}$ \\
\hline 752 & 696 & - & - & - & - & - & $\mathrm{U}$ & - & - & $\mathrm{U}$ \\
\hline 789 & 746 & - & - & -53 & -52 & - & $\mathrm{Y}$ & $-43 \pm 2(14)$ & - & M \\
\hline 913 & 934 & - & - & - & - & - & $\mathrm{N}$ & - & - & $\mathrm{N}$ \\
\hline 1011 & 1095 & - & - & - & - & - & $\mathrm{Y}$ & - & - & M \\
\hline 1054 & 1168 & -49 & - & - & - & - & $\mathrm{Y}$ & - & - & M \\
\hline 1088 & 1211 & -33 & -41 & -31 & - & - & $\mathrm{N}$ & - & $\mathrm{N}$ & $\mathrm{N}$ \\
\hline 1133 & 1270 & - & - & - & -61 & - & $\mathrm{Y}$ & $-55 \pm 3(15)$ & - & M \\
\hline 144 & - & - & - & & & & $\mathrm{U}$ & - & - & $\mathrm{U}$ \\
\hline 459 & - & - & - & -44 & - & - & $\mathrm{U}$ & - & M & M \\
\hline 808 & - & - & - & - & - & - & $\mathrm{U}$ & - & - & $\mathrm{U}$ \\
\hline 1060 & - & - & - & - & - & - & $\mathrm{U}$ & - & - & $\mathrm{U}$ \\
\hline
\end{tabular}

M - McNamara (1980) number.

K - Küstner (1923) number.

$\mathrm{M}, \mathrm{N}, \mathrm{U}$ - denotes the members, non-members and stars with an uncertain membership respectively.

SS70 - Strom \& Strom (1970).

SH84 - Stryker \& Hrivnak (1984).

DS87(CA) - Drilling \& Schönberner (1987), Calar Alto spectra.

DS87(KPNO) - Drilling \& Schönberner (1987), Kitt Peak spectra.

MPMR89 - Manteiga et al. (1989).

ML94 - Milone \& Latham (1994).

SN94 - Schönberner \& Napiwotzki (1994).

* - Based on the study of Milone \& Latham (1994), Gim et al. (1998a) considered the membership of K88 to be uncertain. However, by looking at the original data of Milone \& Latham (Table 2 therein), it became evident that Gim et al. used erroneously the data of K68 instead, which is a radial-velocity non-member.

Mg II 4481 Å-weak stars, and NGC 7789 blue stragglers (see Table 7 for the spectral class assignments for program stars). As can be seen, several of the stragglers resemble the most extreme Mg II $4481 \AA$ A-weak stars. In spite of this, the stragglers have rather low projected rotational velocities (cf. Table 2) as compared with the Mg II $4481 \AA$-weak stars for which a mean of more than $100 \mathrm{~km} \mathrm{~s}^{-1}$ appears to be appropriate (Abt \& Morrell 1995). Interestingly, the two certain non-members, K409 and K677, show virtually solar magnesium abundances, thereby giving additional evidence for their non-membership. $\lambda$ Boo and Vega-like stars. Chemically peculiar A stars of the $\lambda$ Boo type occur at various rotational velocities with a typical $v \sin i$ value of $\approx 100 \mathrm{~km} \mathrm{~s}^{-1}$. Several recent studies of the $\lambda$ Boo-type stars (see, Andrievsky et al. 1998; Paunzen et al. 1999) have shown that in their atmospheres many metals have significantly reduced abundances. In particular, magnesium, calcium and scandium may be depleted by a factor of about 10-100. Similar underabundances are also detected for titanium and iron. At present, there is accumulating evidence that there should be a link between the $\lambda$ Boo phenomenon (extreme abundance 


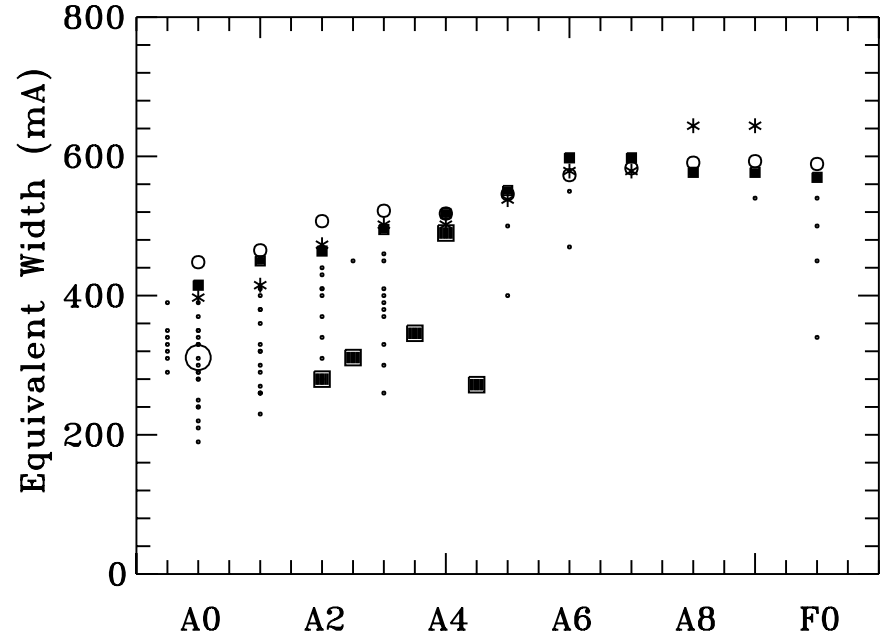

Fig. 4. Equivalent width of the Mg II $4481 \AA$ line vs. spectral class: small circles - class V, small filled squares - class IV, asterisks - class III, large filled squares - program blue stragglers, large open circle - Vega, dots - Mg II $4481 \AA$-weak stars

anomalies) and Vega-like stars characterized by a milder metal deficiency. Dunkin et al. (1997) recently analysed several Vega-like stars and found in some cases relatively strong depletion of magnesium and calcium, while the abundances of iron-group elements are solar within 0.25 dex.

Summarizing, the abundance peculiarities found in the NGC 7789 blue straggler are difficult to attribute to any chemical peculiarity known among A-type stars. This peculiarity is also in contrast with the results reported in our Paper II. None of the straggler stars from three open clusters exhibits, for instance, such a profound magnesium deficiency as found in the present study. The only distinct feature which unites the blue stragglers from the present and previous studies is a low projected rotational velocity.

Concluding this investigation of the blue stragglers in the NGC 7789 field we state that:

a) All the confirmed cluster members show a remarkably superficial magnesium deficiency, while the nonmembers possess a nearly normal abundance;

b) The iron and titanium abundances in all blue stragglers agree with the mean cluster metallicity as derived from red giant stars;

c) The (projected) rotational velocities of the confirmed blue stragglers are significanly lower than normal for their spectral types.

The present study was hampered by the fact that the most powerful telescopes available when the observations were made did not allow us to perform a high-resolution study of the total blue-straggler population of NGC 7789 relative to that cluster's turn-off stars. Such an ambitious project would certainly require the use of 8-10 m class telescopes in order to get spectra of sufficient resolution, signal-tonoise ratio and wavelength coverage. Only then might we
Table 7. Mg II $4481 \AA$ line in program stars

\begin{tabular}{lrcc}
\hline Star & $T_{\text {eff }}(\mathrm{K})$ & $\mathrm{Sp}^{*}$ & $E W(4481)(\mathrm{mA})$ \\
\hline K88 & 8900 & A2-A3 & 320 \\
K316 & 8950 & A2 & 280 \\
K371 & 8500 & A4 & 490 \\
K746 & 8600 & A3-A4 & 353 \\
K1270 & 8300 & A4-A5 & 270 \\
\hline K409 & 9480 & A0 & 640 \\
K677 & 10680 & B9 & 460 \\
K1211 & 12630 & B7-B8 & 280 \\
Vega & 9500 & A0 & 311 \\
\hline * - spectral types are roughly estimated by us between \\
III-V luminosity classes using Lang (1992).
\end{tabular}

be able to answer the fundamental questions on the origin and evolution of blue straggler stars in open clusters.

Acknowledgements. Authors are thankful to the referee, Dr. S. J. Adelman, for his valuable comments. SMA is also grateful to the Astrophysikalisches Institut of Potsdam (Germany) for the financial support and the opportunity to perform this work using its institutional facilities. The necessary information has been obtained through the SIMBAD and VALD data bases.

\section{References}

Abt, H., \& Morrell, N. I. 1995, ApJS, 99, 135

Adelman, S. J. 1999, MNRAS, 310, 146

Adelman, S. J., \& Gulliver, A. F. 1990, ApJ, 348, 712

Ahumada, J., \& Lapasset, E. 1995, A\&AS, 109, 375

Andrievsky, S. M., Chernyshova, I. V., Klochkova, V. G., \& Panchuk, V. E. 1998, CoSka, 27, 446

Andrievsky, S. M., Schönberner, D., \& Drilling, J. 2000, A\&A, 356, 517, Paper II

Breger, M. 1982, ApJ, 263, 199

Caliskan, H., \& Adelman, S. J. 1997, 288, 501

Cayrel, R., Burkhart, C., \& Van't Veer, C. 1991, The photospheric abundance connection, ed. G. Michaud, \& A. V. Tutukov (Kluwer Acad. Publishers), 99

Drilling, J. S., \& Schönberner, D. 1987, IAU Coll. 95, The second conference on faint blue stars, ed. A. G. D. Philips, D. S. Hayes, \& J. Liebert (L. Davis Press), 587

Dunkin, S. K., Barlow, M. J., \& Ryan, S. G. 1997, MNRAS, 286,604

Friel, E. D., \& Janes, K. A. 1993, A\&A, 267, 75

Galazutdinov, G. A. 1992, Prepr. SAO RAS, No. 92

Gim, M., Vandenberg, D. A., Stetson, P. B., Hesser, J. E., \& Zurek, D. R. 1998a, PASP, 110, 1318

Gim, M., Hesser, J. E., McClure, R. D., \& Stetson, P. B. 1998b, PASP, 110, 1172

Hill, G. M. 1995, A\&A, 294, 53

Holweger, H., Gigas, D., Steffen, M. 1986, A\&A, 155, 58

Hubeny, I., Lanz, T., \& Jeffery, C. S. 1994, Newslett. Analysys Astron. Spectra, 20, 30 
Kurucz, R. L. 1991, Precision photometry: Astrophysics of the Galaxy, ed. A. G. D. Philip, A. R. Upgren, \& K. A. Janes (L. Davis Press), 27

Küstner, F. 1923, Veröff. Univ. Sternw. Bonn, No. 18

Lang, K. R. 1992, Astrophysical data (Springer-Verlag)

Manteiga, M., Pickles, A. J., \& Martinez Roger, C. 1989, A\&A, 210,66

Manteiga, M., Martinez Roger, C., Morales, C., \& Sabau, L. 1991, A\&A, 251, 49

McNamara, B. J. 1980, PASP, 92, 682

McNamara, B. J., \& Solomon, S. 1981, A\&AS, 43, 337

Mermilliod, J.-C. 1999, WEBDA database, obswww. unige.ch/webda/

Milone, A., \& Latham, D. W. 1994, AJ, 108, 182
Moon, T. T., \& Dworetsky, M. M. 1985, MNRAS, 217, 305

Napiwotzki, R. 1994, priv. communication

Paunzen, E., Andrievsky, S. M., Chernyshova, I. V., et al. 1999, A\&A, 351, 981

Pendl, E. S. 1975, A\&A, 41, 239

Pilachowski, C. A. 1985, PASP, 97, 801

Saio, H., \& Wheeler, J. G. 1980, ApJ, 242, 1176

Sadakane, K., \& Nishimura, M. 1981, PASJ, 33, 189

Schönberner, D., \& Napiwotzki, R. 1994, A\&A, 282, 106

Strom, K. M., \& Strom, S. E. 1970, ApJ, 162, 523

Stryker, L. L., \& Hrivnak, B. J. 1984, ApJ, 278, 215

Tiede, G. P., Martini, P., \& Frogel, J. A. 1997, AJ, 114, 694

Twarog B. A., \& Tyson, N. 1985, AJ, 90, 124

Vallenari, A., Carraro, G., \& Richichi, A. 2000, A\&A, 353, 147 